\title{
MICRONUTRIENTES NO CRESCIMENTO E DESENVOLVIMENTO INFANTIL
}

\section{MICRONUTRIENTS IN CHILD GROWTH AND DEVELOPMENT}

\section{Dixis Figueroa Pedraza ${ }^{1}$ \\ Daiane de Queiroz ${ }^{2}$}

Figueroa Pedraza D; Queiroz D. Micronutrientes no crescimento e desenvolvimento infantil. Rev. Bras. Cresc. e Desenv. Hum. 2011; 21(1): 156-171.

\section{Resumo}

Objetivo: abordar a importância dos micronutrientes zinco, ferro e vitamina A no crescimento linear e desenvolvimento infantil. Método: foram consultadas as bases de dados Medline e LILACS utilizando duas estratégias de busca: i) growth AND zinc AND iron AND vitamin AAND child, preschool OR infant, para o tema micronutrientes e crescimento; e ii) child development AND zinc AND iron AND vitamin A, para o tema micronutrientes e desenvolvimento. Os artigos foram classificados quanto ao tipo de manuscrito e principais resultados. Resultados: foram incluídos 19 artigos referentes ao crescimento e 5 artigos referentes ao desenvolvimento. Discussão: considerando as prevalências, sugere-se que as deficiências de ferro e zinco devem contribuir, concurrentemente, com o déficit de estatura. O papel do zinco no sistema hormonal primário justifica ser o nutriente de maior importância no crescimento, afetando a estatura das crianças tanto em estados de deficiência leve como moderada. Evidências fisiológicas e experimentais colocam a importância dos micronutrientes ferro e zinco no desenvolvimento e déficit cognitivo nos primeiros anos da vida. Apesar disso, os resultados ainda não são conclusivos. Pensa-se que o zinco pode limitar processos neuropsicológicos específicos, já o ferro é essencial para o funcionamento do sistema neuro-transmissor. Estudar o efeito das deficiências de micronutrientes no desenvolvimento apresenta vários problemas metodológicos e analíticos. O crescimento e desenvolvimento representam um processo com determinação comum no qual a deficiência de zinco pode representar a forma mais comum em que o crescimento linear poderia ser utilizado como um bom preditor de desenvolvimento cognitivo.

Palavras-chave: micronutrientes; crescimento; desenvolvimento infantil; desenvolvimento cognitivo; zinco; ferro; vitamina A.

1 Doutor em Nutrição. Programa de Pós-Graduação em Saúde Pública e Núcleo de Estudos e Pesquisas Epidemiológicas da Universidade Estadual da Paraíba.

2 Mestranda do Programa de Pós-graduação em Saúde Pública da Universidade Estadual da Paraíba.

Correspondência para: Dixis Figueroa Pedraza - Universidade Estadual da Paraíba - Núcleo de Estudos e Pesquisas Epidemiológicas - Pró-Reitoria de Pós-Graduação e Pesquisa (PRPGP) Av. das Baraúnas, 351 - Campus Universitário - Bairro Bodocong - Campina Grande, Paraíba - CEP: 58109-753 - Telefone: (83) 3315-3415 - FAX: (83) 3315-3388 - E-mail: dixisfigueroa@gmail.com 


\begin{abstract}
Objective: the present work addresses the importance of micronutrients zinc, iron and vitamin A in linear growth and child development. Method: we consulted the databases of Medline and LILACS. We used two search strategy: i) growth AND zinc AND iron AND vitamin A AND child, preschool OR infant, for micronutrients and growth; and ii) child development AND zinc AND iron AND vitamin A, for micronutrients and development. The articles were classified according to type and principal results. Results: we included 19 articles concerning the growth and 5 articles relating to development. Discussion: considering the prevalence, it is suggested that deficiencies of iron and zinc should contribute, concurrently with the stunting. The role of zinc in the primary hormonal system justifies it being the nutrient of greatest importance in growth, affecting children's height both in light or moderate states of deficiency. Physiological and experimental evidences pose the importance of iron and zinc micronutrients in development and cognitive deficit in the first years of life. Besides that, the results are still not conclusive. It is thought that zinc can limit specific neuropsychological processes, now iron is essential for the neuro-transmitter system operation. Studing the isolated effect of micronutrient deficiencies in development presents several methodological and analytical problems. Growth and development represents a process with common determination in which zinc deficiency may represent the most common way in which the linear growth could be used as a good predictor of cognitive development.
\end{abstract}

Key words: micronutrients; growth; child development; cognitive development; zinc; iron; vitamin A.

\section{INTRODUÇÃO}

O crescimento é um processo dinâmico, contínuo, regular e extremamente organizado que tem início com a fecundação (princípio da vida), sendo expresso através do aumento do tamanho corporal. Quanto ao seu prolongamento, os autores o marcam pelo crescimento linear que acontece até os 20 anos nos homens e até os 18 anos nas mulheres. $\mathrm{O}$ crescimento supõe um aumento, a velocidades diferentes, do número (hiperplasia) e/ou do tamanho (hipertrofia) de suas células. Do ponto de vista sistêmico, é controlado, regulado e atualizado pela informação contida nos genes, caracterizando-se nos primeiros anos de vida por uma dinâmica de troca, compensação e adaptação $0^{1,2,3}$.

$\mathrm{Na}$ medição do crescimento infantil, prioriza-se a utilização dos índices peso ao nascer, peso por idade, peso por estatura e estatu- ra por idade. O peso do recém-nascido constitui um importante indicador do crescimento intrauterino. O peso como medida de crescimento mede os incrementos (ou diminuições) da massa corporal por hiperplasia e/ou por hipertrofia. Por sua vez, a estatura é uma medida corporal que expressa a dimensão longitudinal ou linear do corpo humano. Para que o peso e a estatura se transformem em índices do crescimento, é preciso relaciona-los a outras variáveis que a eles estão intimamente associados. O índice peso por idade possibilita, principalmente, diagnosticar as alterações do crescimento ponderal. $\mathrm{O}$ índice peso por estatura expressa a harmonia do crescimento, refletindo a perda ou excesso de massa corporal indicativa de um processo de desnutrição aguda ou de sobrepeso. O índice estatura por idade expressa deficiências nutricionais de longa duração ou os efeitos cumulativos dos múltiplos fatores adversos à saúde 3 . 
As evidências científicas indicam a importância de prestar maior atenção ao crescimento linear durante os primeiros três anos de vida, pois a recuperação do déficit de estatura é limitada após o segundo ano de vida e pode se estender até a vida adulta e a gerações futuras. Essas situações são mais marcantes em condições de pobreza. A estatura constitui um fator de grande importância para o desenvolvimento cognitivo, o rendimento produtivo, o estado de saúde e o desenvolvimento econômico ${ }^{4,5}$.

Define-se como desenvolvimento a capacidade progressiva do ser humano em realizar funções cada vez mais complexas. Este processo é o resultado da interação entre os fatores biológicos, próprios da espécie e do indivíduo, e os fatores culturais, próprios do meio social em que o indivíduo está inserido. Assim, a aquisição de novas habilidades está diretamente relacionada, não apenas à faixa etária da criança, mas também às interações vividas com os outros seres humanos do seu grupo social.

Didaticamente, o desenvolvimento é descrito de acordo com o domínio progressivo de algumas funções que indicam aperfeiçoamento: desenvolvimento sensorial, principalmente da audição e visão; habilidades motoras grosseiras, referentes à utilização dos grandes músculos do corpo; habilidades motoras finas, relacionadas ao uso dos pequenos músculos das mãos; desenvolvimento da linguagem; desenvolvimento social, emocional e cognitivo, referidos aos processos mentais superiores como as capacidades de pensar, de memorizar e de aprender ${ }^{2,6}$.

Contrário ao crescimento, o desenvolvimento não pode ser medido com um mesmo indicador ao decorrer da vida. Dessa maneira, a avaliação do desenvolvimento implica a observação das funções neurológicas, cognitivas, afetivas e sociais. O objetivo é tornar possível a identificação de situações da vida que indiquem alterações na maturação ou aperfeiçoamento dessas funções. Várias são as pro- postas e modelos para a avaliação do desenvolvimento, destacando a aplicação de testes ou escalas, exames e outros procedimentos. Os exames e outros procedimentos similares baseiam-se em técnicas de observação de algumas posturas, comportamentos e reflexos presentes em determinadas faixas etárias da criança. Os testes ou escalas de desenvolvimento devem ser validados, destacando as seguintes: Bayley, 1993; Frankenburg e Dodds, 1967; Gesell e Amatruda, 1945; Pinto, Vilanova e Vieira, $1997^{6,7}$.

Apesar das diferenças conceituais existentes sobre crescimento e desenvolvimento, as investigações científicas sobre o assunto atestam que são processos simultâneos e diferenciados, que têm determinações comuns, ou que a maior parte dos determinantes que atuam sobre o crescimento, também atuam sobre o desenvolvimento ${ }^{3}$. Nesse contexto, é de destacar que os três primeiros anos da vida representam o período mais importante. Essa fase da vida constitui a de maior vulnerabilidade relacionada ao aparecimento de doenças infecciosas, de desnutrição e de alterações significativas no sistema nervoso central. De caráter positivo é o fato de ser a etapa do ciclo da vida com maiores possibilidades de reversão de danos causados pelas doenças e as deficiências nutricionais, inclusive de micronutrientes ${ }^{4,8}$.

$\mathrm{Na}$ maioria dos países em desenvolvimento, e também em alguns grupos populacionais de países desenvolvidos, a alimentação habitual é insuficiente para suprir $100 \%$ dos requerimentos de micronutrientes das crianças, principalmente, para os minerais ferro, zinco e cálcio. Isso também é válido, embora em menor grau, para algumas vitaminas, incluindo a vitamina A. Este fato alerta para a necessidade da administração de suplementos nutricionais para otimizar o potencial genético de crescimento físico, além do desenvolvimento, assim como prevenir o surgimento de doenças infecciosas. Assim, as políticas e programas devem direcionar esforços para a 
melhoria do estado nutricional de micronutrientes de crianças na faixa etária pré-escolar?.

Desta forma, o objetivo é descrever a função dos micronutrientes no crescimento e desenvolvimento infantil.

\section{MÉTODO}

O artigo foi desenvolvido com a consulta de literatura básica e a partir das evidências de estudos observacionais e experimentais. Aprofundam-se as consequências das deficiências de vitamina A, ferro e zinco no crescimento linear e no desenvolvimento cognitivo com base em revisões sistemáticas sobre o tema.

Foram consultadas as bases de dados: LILACS (Literatura Latino-Americana e do Caribe em Ciências da Saúde) e MEDLINE/ PUBMED (US National Library of Medicine's - NLM). As consultas incluíram os artigos publicados nos últimos dez anos que envolveram seres humanos e nos idiomas inglês, português e espanhol.

A busca bibliográfica, realizada em julho de 2010 por dois revisores, utilizou as seguintes estratégias:

- Primeira estratégia de busca (micronutrientes e crescimento infantil): utilizaram-se os termos de busca growth AND zinc AND iron AND vitamin A AND child, preschool OR infant

- Segunda estratégia de busca (micronutrientes e desenvolvimento infantil): utilizaram-se os termos de busca child development AND zinc AND iron AND vitamin A

Os estudos identificados foram classificados como excluídos e incluídos considerando os critérios estabelecidos para esses fins:

- critérios de inclusão: artigos de revisão sistemática, estudos realizados em crianças pré-escolares, estudos realizados em crianças sadias, estu- dos com análises de associação entre as deficiências de micronutrientes e o crescimento ou desenvolvimento infantil;

- critérios de exclusão: artigos de revisão narrativa, estudos realizados em grupo etário diferente ao de crianças pré-escolares (crianças maiores de 5 anos, adolescentes, adultos, mulheres grávidas), estudos realizados em crianças com algum tipo de patologia, estudos em que não foi analisada a associação entre as deficiências de micronutrientes e o crescimento ou desenvolvimento infantil.

As informações selecionadas nos artigos para sua caracterização foram: autor e ano de publicação, tipo de manuscrito (revisão, observacional ou experimental) e principais resultados considerando o objetivo deste trabalho.

\section{RESULTADOS}

Para a primeira estratégia de busca foram identificados 49 artigos (todos no MEDLINE), dos quais 30 foram excluídos, destes, 12 eram artigos de revisão, 9 não apresentaram resultados de associação entre micronutrientes e crescimento infantil, 5 estudos foram realizados em grupo etário diferente ao de crianças pré-escolares e 4 estudos realizados em crianças com algum tipo de doença. Para a segunda estratégia de busca foram identificados 30 artigos (26 no MEDLINE e 4 no LILACS), dos quais 25 foram excluídos, destes 10 eram artigos de revisão, 14 não apresentaram resultados de associação entre micronutrientes e desenvolvimento infantil e 1 estudo realizado em crianças com algum tipo de doença. Ao final foram incluídos 19 arti$\operatorname{gos}^{8,10-27}$ referentes ao crescimento e 5 arti$\operatorname{gos}^{8,10,28-30}$ referentes ao desenvolvimento. A 
tabela 1 mostra a distribuição dos artigos incluídos segundo o tipo de manuscrito e base de dados no qual foi identificado.

As tabelas 2 e 3 apresentam uma síntese dos principais resultados dos manuscritos enfocando a importância dos micronutrientes zinco, ferro e vitamina A no crescimento e desenvolvimento infantil.

\section{DISCUSSÃO}

\section{Principais funções biológicas dos micronu- trientes ferro, vitamina $A$ e zinco}

A saúde e o bem-estar das crianças dependem da interação entre seu potencial genético e fatores externos como adequação nutricional, ambiente saudável, interação social e cuidados adequados. A nutrição tem um papel muito importante na promoção do crescimento físico, no desenvolvimento neuropsicológico e no combate às doenças infecciosas que afetam, principalmente, as crianças ${ }^{8}$.
Devido aos avanços no controle da desnutrição energético-protéica, as deficiências de micronutrientes adquirem cada vez maior relevância enquanto problema de saúde pública. As deficiências de vitamina A, ferro e zinco ainda apresentam altas prevalências na maioria dos países em desenvolvimento, ocasionando diversos agravos à saúde dos indivíduos. Esses nutrientes são essenciais para o adequado funcionamento do organismo e para a otimização do processo de crescimento e desenvolvimento ${ }^{8,30}$.

A vitamina A é um micronutriente importante para diversos processos metabólicos, tendo papéis fisiológicos muito diversificados, atuando no bom funcionamento do processo visual, na diferenciação celular, na integridade do tecido epitelial, na reprodução e no sistema imunológico. Apresenta especial importância durante os períodos de proliferação e de rápida diferenciação celular tais como a gestação, o período neonatal e a infância ${ }^{30,31}$.

O ferro é o oligoelemento mais abundante no organismo humano. Participa de di-

Tabela 1: Distribuição dos manuscritos nas bases de dados MEDLINE/PUBMED e LILACS

\begin{tabular}{lccc}
\hline \multicolumn{1}{c}{ Tipo de manuscrito } & \multicolumn{3}{c}{ Base de dados } \\
\hline & LILACS & MEDLINE/PUBMED & TOTAL \\
\hline Primeira estratégia de busca & 0 & 9 & 9 \\
- Experimental & 0 & 3 & 3 \\
- Observacional & 0 & 19 & 7 \\
- Revisão sistemática & 0 & & 19 \\
\hline Total & & 2 & 2 \\
\hline Segunda estratégia de busca & 0 & 0 & 0 \\
- Experimental & 0 & 2 & 2 \\
- Observacional & 1 & 4 & 4 \\
- Revisão sistemática & & & \\
\hline Total & 1 & & \\
\hline
\end{tabular}

LILACS: Literatura Latino-Americana e do Caribe em Ciências da Saúde MEDLINE: Medical Literature Analysis and Retrieval System Online 
Tabela 2. Síntese dos manuscritos que versaram sobre micronutrientes e crescimento infantil nas bases de dados LILACS e MEDLINE/PUBMED.

\begin{tabular}{ll}
\hline Autor, ano & Tipo de manuscrito \\
\hline $\begin{array}{l}\text { Allen LH et al }{ }^{10}, \\
2009\end{array}$ & Revisão sistemática \\
Ramakrishnan U & Experimental \\
et a1 ${ }^{11}, 2009$ & \\
Ramakrishnan U & Revisão sistemática \\
et al12, 2009 & \\
Bhutta ZA et al ${ }^{13}$, & Revisão sistemática \\
2008 & \\
Chen K et al ${ }^{14}$, & Experimental \\
2008
\end{tabular}

Lutter CK et $\mathrm{al}^{15}$, Observacional 2008 Principais resultados

Suplementação com múltiplos micronutrientes resulta em pequena, mas significativa melhora no comprimento das crianças em comparação ao grupo controle.

Crianças suplementadas com múltiplos micronutrientes tiveram maior comprimento quando comparadas ao grupo controle.

A suplementação múltipla de micronutrientes melhora o crescimento linear de crianças.

A suplementação alimentar, em populações com alimentação insuficiente, aumenta o escore $\mathrm{z}$ do índice altura/idade em 0,41 .

O crescimento linear, comparando-se a primeira e a última medida de cada grupo, foi significativamente maior para os três grupos de crianças suplementadas: grupo 1 (vitamina A), grupo 2 (vitamina A e ferro) e grupo 3 (vitamina A, ferro, tiamina, riboflavina, ácido fólico, niacina, zinco e cálcio).

A suplementação alimentar teve impacto positivo e significativo para o índice altura/idade quando comparado ao grupo controle.

Uauy $\mathrm{R}$ et $\mathrm{al}^{16}$, Revisão sistemática 2008

As deficiências de micronutrientes (ferro, vitamina A e zinco) coexistem com o déficit de estatura e a obesidade. Estratégias de intervenção para prevenir a má nutrição devem enfatizar as melhoras no crescimento linear nos primeiros dois a três anos de vida, mais do que se preocupar com o ganho de peso.

Elizabeth KE et Observacional $\mathrm{al}^{17}, 2007$

Recém-nascidos com baixo peso ao nascer (pré-termo e a termo) tiveram reservas de nutrientes significativamente menores quando comparados aos bebês a termo de peso adequado. Infecções recorrentes e hábitos alimentares inadequados podem afetar ainda mais as reservas de nutrientes, implicando a necessidade de suplementação.

Fahmida $\mathrm{U}$ et $\mathrm{al}^{18}$, Experimental 2007

A suplementação com zinco teve um efeito positivo sobre o crescimento nos casos de baixa concentração de hemoglobina/ferro corrigida.

Katz J et al ${ }^{19}, 2006$ Experimental

O peso ao nascer de crianças cujas mães foram suplementadas (ácido fólico + ferro + vitamina A ou ácido fólico + ferro + zinco + vitamina A ou micronutrientes múltiplos) foi significativamente maior do que nas crianças cujas mães foram suplementadas somente com vitamina A. 


\begin{tabular}{lll}
\hline Autor, ano & Tipo de manuscrito & Principais resultados \\
\hline Hop le $\mathrm{T}$ et $\mathrm{al}^{20}$, Experimental & & O escore $\mathrm{z}$ do índice altura/idade piorou significativamente \\
2005 & em todos os grupos de crianças suplementadas: grupo 1 \\
& (múltiplos micronutrientes diariamente), grupo 2 (múltiplos \\
& micronutrientes semanalmente), grupo 3 (ferro diariamen- \\
& te), grupo 4 (placebo diariamente). A diminuição do escore \\
& $\mathrm{z}$ foi significativamente menor no grupo 1 quando compa- \\
& rado ao grupo 2 e 3.
\end{tabular}

Smuts $\mathrm{CM}$ et $\mathrm{al}^{21}$, Experimental 2005

Untoro $\mathrm{J}$ et $\mathrm{al}^{22}$, Experimental 2005

Singh M8, 2004 Revisão sistemática

Christian $\mathrm{P}$ el $\mathrm{al}^{23}$, Experimental 2003

Oelofse $\mathrm{A}$ et $\mathrm{al}^{24}$, Experimental 2003

Rivera JA et $\mathrm{al}^{25}$, Revisão sistemática 2003

Bhandari $\mathrm{N}$ et $\mathrm{al}^{26}$, Revisão sistemática 2001

Dijkhuizen MA et Observacional $\mathrm{al}^{27}, 2001$
Não houve diferença no crescimento linear entre o grupo placebo e os grupos de crianças suplementadas: grupo 1 (múltiplos micronutrientes diariamente), grupo 2 (múltiplos nutrientes $1 \mathrm{v} /$ semana), grupo 3 (ferro diariamente).

Não houve diferença no crescimento linear entre o grupo placebo e os grupos de crianças suplementadas: grupo 1 (múltiplos micronutrientes diariamente), grupo 2 (múltiplos micronutrientes $2 \mathrm{v}$ /dia uma vez por semana), grupo 3 (ferro diariamente).

A deficiência de micronutrientes acontecem normalmente de forma combinada, sendo muitos deles de grande importância para o alcance do potencial genético de crescimento, principalmente nos três primeiros anos de vida que constitui a etapa de maior vulnerabilidade.

Mulheres grávidas suplementadas com múltiplos micronutrientes não apresentaram qualquer benefício adicional na redução do baixo peso ao nascer quando comparadas às suplementadas com ácido fólico e ferro.

Não houve diferença no crescimento linear entre o grupo de crianças que receberam alimentos complementares enriquecidos com micronutrientes e o grupo controle.

As deficiências de vitamina A e ferro afetam o crescimento linear somente em condições de deficiência severa. O contrário é observado na deficiência de zinco, considerada a causa mais comum do déficit de estatura em crianças tanto nos casos de deficiência severa, leve ou moderada.

A suplementação com zinco tem um modesto, mas significativo impacto no crescimento linear em crianças pré-escolares. O benefício da suplementação com ferro no crescimento linear parece provável somente em crianças anêmicas. A suplementação com vitamina $\mathrm{A}$, provavelmente, não tem um importante efeito no crescimento linear.

Nenhuma associação foi encontrada entre os indicadores do estado nutricional de micronutrientes e o crescimento linear. 
Tabela 3: Síntese dos manuscritos que versaram sobre micronutrientes e desenvolvimento infantil nas bases de dados LILACS e MEDLINE/PUBMED

\begin{tabular}{|c|c|c|}
\hline Autor, ano & Tipo de manuscrito & Principais resultados \\
\hline $\begin{array}{l}\text { Allen LH et } \mathrm{al}^{10} \text {, } \\
2009\end{array}$ & Revisão sistemática & $\begin{array}{l}\text { Proporcionar suplementação com múltiplos micronutrientes } \\
\text { melhora o desenvolvimento motor de crianças jovens quando } \\
\text { comparadas com a suplementação de um ou dois } \\
\text { micronutrientes. }\end{array}$ \\
\hline $\begin{array}{l}\text { Wehby GL et } \mathrm{al}^{28} \text {, } \\
2008\end{array}$ & Experimental & $\begin{array}{l}\text { O uso de suplementação com zinco no pré-natal foi } \\
\text { associado com maior risco no déficit de desenvolvimento } \\
\text { de crianças brancas. O uso de suplementação com vitamina } \\
\text { A foi associada com diminuição no risco do déficit de } \\
\text { desenvolvimento de crianças brancas. }\end{array}$ \\
\hline $\begin{array}{l}\text { Silva LSV et } \mathrm{al}^{29} \text {, } \\
2007\end{array}$ & Revisão sistemática & $\begin{array}{l}\text { A carência materna de ferro durante o período gestacional } \\
\text { pode comprometer o desenvolvimento do cérebro do recém- } \\
\text { nascido, ocasionando diminuição da capacidade cognitiva, } \\
\text { aprendizagem, concentração, memorização e alteração do } \\
\text { estado emocional. }\end{array}$ \\
\hline Singh $\mathrm{M}^{8}, 2004$ & Revisão sistemática & $\begin{array}{l}\text { A deficiência de micronutrientes acontecem normalmente } \\
\text { de forma combinada, sendo muitos deles de grande } \\
\text { importância para o alcance do potencial genético de } \\
\text { desenvolvimento mental, principalmente nos } 3 \text { primeiros } \\
\text { anos de vida que constitui a etapa de maior vulnerabilidade. }\end{array}$ \\
\hline $\begin{array}{l}\text { Oelofse A et } \mathrm{al}^{24} \text {, } \\
2003\end{array}$ & Experimental & $\begin{array}{l}\text { Não foi observada diferença no escore do desenvolvimento } \\
\text { psicomotor entre o grupo de crianças que ingeriram } \\
\text { alimentos complementares enriquecidos com } \\
\text { micronutrientes e o grupo de crianças que não consumiram } \\
\text { esses alimentos. }\end{array}$ \\
\hline
\end{tabular}

versos processos metabólicos, incluindo o transporte de elétrons e oxigênio, o metabolismo de catecolaminas (co-fator da enzima tirosina hidroxilase) e a síntese de DNA. O ferro também é um componente essencial da hemoglobina, da mioglobina, da ferritina e de outras metaloenzimas, que são necessárias para a função celular normal. É de destacar, ainda, o papel do ferro no sistema imune ${ }^{30,32,33}$.

$\mathrm{O}$ zinco cumpre numerosas funções estruturais, bioquímicas e de regulação. Depois do ferro, o zinco é o micromineral com distribuição mais abundante no corpo humano, encontrando-se em grandes quantidades em todos os tecidos. Apresenta especial importância em diversos processos biológicos do organis- mo, incluindo a síntese protéica, a síntese dos ácidos nucléicos (DNA e RNA) e o metabolismo energético de carboidratos e lipídios. Entre as principais funções do zinco, destaca-se seu papel enzimático, seja na ativação catalítica de diversos processos enzimáticos nas principais vias metabólicas do organismo, ou na estrutura e regulação enzimática. $\mathrm{O}$ zinco também é um micronutriente necessário à reprodução e maturação, na regulação hormonal da divisão celular, no reparo de tecidos, na resposta imune e no funcionamento das membranas celulares ${ }^{34,35}$.

A deficiência de micronutrientes está relacionada com uma série de efeitos deletérios na infância, com consequente aumento das 
taxas de morbi-mortalidade, dentre outros agravos à saúde. O potencial genético das crianças, em relação ao crescimento físico, pode estar comprometido pela deficiência subclínica de micronutrientes. Neste contexto, o zinco, o ferro e a vitamina A merecem atenção especial, pois são os nutrientes que mais limitam o crescimento e desenvolvimento infantil, representam os estados deficitários mais comuns e apresentam importantes inter-relações metabólicas ${ }^{8,12,36}$.

\section{Micronutrientes e crescimento linear}

A primeira manifestação vital de um crescimento inadequado é nascer com baixo peso. Este fato representa um alto risco de mortalidade durante o período neonatal e um aumento na probabilidade de ter baixo peso ou baixa estatura nos primeiros anos de vida. $\mathrm{O}$ baixo peso ao nascer (BPN), definido como o peso no nascimento menor de 2500 gramas, tem como principais determinantes o nascimento pré-termo e a Restrição do Crescimento Intrauterino (RCIU). Consideram-se prematuras as crianças que nascem em idade gestacional menor de 37 semanas. A RCIU é estabelecida para definir as crianças que nascem com peso ao nascer inferior ao percentil 10 segundo a idade gestacional. As vitaminas e minerais são micronutrientes de grande importância na saúde de mulheres grávidas e no crescimento fetal. Em sentido geral, é considerado que as deficiências de zinco e vitamina A constituem fatores de risco nutricionais para RCIU, e que as deficiências de zinco e ferro são fatores de risco nutricionais para o nascimento pré-termo ${ }^{37}$.

O déficit de estatura infantil representa um problema nutricional multicausal, no qual os fatores alimentares interagem com os problemas de saúde num contexto de condições socioeconômicas desfavoráveis. Assim, a variabilidade encontrada na estatura de crianças pode ser interpretada de maneira diferente, dependendo do lugar de residência e nível so- cioeconômico. Enquanto nos paises desenvolvidos, essa variabilidade reflete principalmente a influência de fatores hereditários, nos paises em desenvolvimento deve ser interpretada como o efeito de condições ambientais, em especial de alimentação e doenças que afetam o crescimento e desenvolvimento ${ }^{4}$.

Estudos recentes, abordando o déficit de crescimento linear em crianças, destacam a deficiência de micronutrientes como fator de risco de grandíssima importância, sendo o zinco, a vitamina A e o ferro os de maior impacto $^{38}$. O papel desses micronutrientes tem sido claramente demonstrado através de estudos de suplementação com um único micronutriente. Considera-se que as deficiências de ferro $^{26} \mathrm{e}$ vitamina $\mathrm{A}^{39}$ afetam o crescimento somente em condições de deficiência severa $(\mathrm{Hb}<9,5 \mathrm{~g} /$ $\mathrm{dL}$, retinol sérico $<0,35 \mu \mathrm{mol} / \mathrm{L}$ ), enquanto até mesmo a deficiência leve de zinco pode causar prejuízos no crescimento ${ }^{40}$.

Os achados anteriores são consistentes com os conhecimentos sobre as funções metabólicas e fisiológicas do zinco que indicam seu efeito direto no sistema hormonal primário (IGF-I/GH), encarregado de controlar o crescimento na fase pós-natal que é quando acontece o maior crescimento linear. $\mathrm{O}$ ferro e a vitamina A não afetam este sistema diretamente, exercendo o maior efeito nos casos de estoques funcionais esgotados e/ou quando suas deficiências resultam em um incremento da morbidade ${ }^{25}$.

Como as prevalências de níveis baixos de vitamina A (retinol sérico $<0,70 \mu \mathrm{mol} / \mathrm{L}$ ) são inferiores a $20 \%$, para quase todos os países da América Latina, pensa-se que a prevalência de deficiência severa de vitamina A seja ainda menor e, consequentemente, a deficiência da vitamina deva ter uma contribuição mínima para o déficit de estatura na região. No caso do ferro, considerando a prevalência generalizada de anemia $(\mathrm{Hb}<11,0 \mathrm{~g} / \mathrm{dL})$ na América Latina, com poucas diferenças entre as sub-regiões, pensa-se na possibilidade de que 
a anemia ferropriva contribua de maneira importante para o déficit de estatura na região. Dos 15 países que reportam prevalência de anemia $>12 \%, 14$ possivelmente estão em risco médio a moderado de deficiência de zinco, sugerindo que as duas deficiências clínicas devem contribuir, concorrentemente, nas prevalências de desnutrição crônica na região ${ }^{25,38,41}$.

Existem evidências sobre o efeito benéfico da suplementação com micronutrientes no crescimento linear infantil. Os estudos de suplementação com ferro apontam o efeito favorável nos casos de crianças anêmicas. Os conhecimentos atuais indicam que a suplementação com vitamina A tem pouco beneficio no crescimento linear, sendo que alguns dados sugerem impacto nas crianças com deficiência clinica ou bioquímica, mas isso precisa ser confirmado. Os estudos de suplementação com zinco confirmam que este micronutriente tem módico, mas significativo, impacto na estatura, mostrando-se de grande efetividade nas crianças com déficit de estatura ${ }^{25,26}$.

Alguns trabalhos apontam maior efetividade da suplementação múltipla de micronutrientes quando comparada à suplementação com um único micronutriente. Não obstante, os resultados existentes não são conclusivos. Embora alguns estudos demonstrem o efeito positivo da suplementação múltipla com micronutrientes no crescimento em populações específicas, não está estabelecida a efetividade quando os esquemas são aplicados em nível nacional. Por outro lado, não existem estudos que comprovem o custo-efetividade da suplementação com um único micronutriente, principalmente de zinco, estabelecendo a devida comparação com a suplementação múltipla. Acredita-se que a maioria dos casos de déficit de estatura esteja associada com a deficiência concomitante de vários micronutrientes. Supõe-se que, nesses casos, o efeito da suplementação com um único micronutriente no crescimento linear não deve ser significativo. Porém, quando o déficit de estatura associa-se, principalmente, a um micronutriente específico, é provável que a melhor estratégia do ponto de vista de custo-efetividade seja a suplementação do micronutriente deficitário ${ }^{12}$, 25,38 .

\section{Micronutrientes e desenvolvimento cognitivo}

$\mathrm{Na}$ trajetória do desenvolvimento da criança, mesmo antes do nascimento, esta pode ser exposta a múltiplos e contínuos eventos adversos que podem colocar em risco seu desenvolvimento pleno e sadio. Esses eventos adversos constituem-se como fatores de risco que, normalmente, ocorrem em conjunto, potencializando a gravidade de suas consequências. Esses fatores incluem doenças maternas, inadequados estilos de vida durante a gravidez, fatores teratogênicos e tóxicos, má nutrição materna, doenças e traumas vivenciadas pelas crianças, condições socioeconômicas desfavoráveis, carências nutricionais ${ }^{42,46}$.

Devido aos processos de mielinização e diferenciação neuronal, o sistema nervoso central apresenta uma intensa evolução dinâmica de suas estruturas nos primeiros anos de vida. A fase que compreende o período pré-natal, mais especificamente o último trimestre de gestação, e os primeiros anos de vida representa um dos períodos críticos mais importantes para o desenvolvimento cerebral. Essa etapa da vida caracteriza-se por uma grande vulnerabilidade a vários tipos de agressões, inclusive a nutricional, e por ser o período de crescimento mais rápido do cérebro ${ }^{44,45}$.

Estudos com animais de experimentação têm indicado possíveis mecanismos explicativos sobre a influência da deficiência de ferro no crescimento e desenvolvimento cerebral. Em certas áreas cerebrais (substância negra, globus pallidus, núcleos caudado e rubro e putamen) existem altas concentrações 
de ferro. No nível do sistema nervoso central o ferro tem uma função importante na maturação neuronal, na mielinização e na constituição de algumas enzimas envolvidas na síntese, na função e na degradação de neurotransmissores cerebrais. Esses neurotransmissores, designadamente a dopamina, a adrenalina e a serotonina estão envolvidos no controlo da atenção e na modulação comportamenta ${ }^{46,47}$.

Estudos em crianças entre seis e 24 meses de idade com anemia por deficiência de ferro mostram que a deficiência de ferro representa um maior risco de pobre desenvolvimento cognitivo, neuropsicológico, motor e socioemocional, com consequências em curto e longo prazo ${ }^{44,48,49,50}$. Porém, os resultados de estudos clínicos sobre os efeitos da suplementação com ferro no desenvolvimento mental são conflitantes.

Os dados de duas revisões, sendo uma bibliográfica $^{50}$ e uma sistemática ${ }^{44}$, indicam que a suplementação com ferro melhora os escores de desenvolvimento mental de forma modesta. Adicionalmente, coloca-se o efeito mais marcado e significativo nas crianças anêmicas, relacionando-se ainda à severidade e duração da anemia. Os artigos também discutem a influência da idade da criança. Crianças anêmicas de dois anos ou mais suplementadas com ferro, apresentam um claro efeito positivo no desenvolvimento mental/cognitivo. Nas crianças menores de dois anos as evidências são incertas e confusas, pois $\mathrm{S} \sim$ so poucos os estudos com desenhos controlados randomizados desenvolvidos. Além disso, existe maior dificuldade para avaliar o desenvolvimento mental/cognitivo nessa faixa etária. Em relação ao desenvolvimento motor, os estudos apontam falta de associação, mesmo em crianças anêmicas.

Existem provas sobre a associação entre os processos infecciosos e o desenvolvimento da criança. Assim, as deficiências nutricionais podem afetar, direta ou indiretamente, o desenvolvimento e o comportamento da crian- ça através do aumento da morbidade. É por isso que a deficiência de vitamina A pode ser adicionada à lista de deficiências que podem afetar o desenvolvimento infanti1 ${ }^{47,50}$.

Estudos abordando o efeito da suplementação com vitamina A no desenvolvimento infantil são incipientes. Pesquisa realizada na Indonésia ${ }^{51}$, com crianças aos três anos de idade depois de suplementadas no período neonatal com vitamina $\mathrm{A}$, indicou um pequeno efeito benéfico nas pontuações de desenvolvimento, sendo significativo para parâmetros comportamentais. Ao mesmo tempo, observou-se efeito positivo relacionado a sinais e sintomas característicos de doenças infecciosas.

$\mathrm{O}$ zinco é um elemento estrutural e funcional de grande importância para o cérebro. No nível do sistema nervoso central atua na síntese de proteínas importantes para a produção de neurotransmissores e favorece a afinidade para os seus receptores. Destaca-se também a importância do zinco na síntese dos ácidos nucléicos ${ }^{47,52}$. A deficiência de zinco retarda o crescimento e maturação dos neurônios, afetando, também, a atividade eletro fisiológica e de transmissão no cérebro mediante mecanismos não muito bem identificados ${ }^{53,54}$. Acredita-se que o zinco, junto com o cálcio, o potássio e o sódio, tem importância como um modulador chave na excitabilidade neuronal ${ }^{55}$.

A sugestão de que a deficiência de zinco pode conduzir a retardo do desenvolvimento cognitivo está baseada tanto em estudos com animais de experimentação como em estudos envolvendo seres humanos. As pesquisas com animais de experimentação têm mostrado as consequências da deficiência de zinco no desenvolvimento do cérebro, nos níveis de atividade e atenção, na memória e no desenvolvimento cognitivo ${ }^{50}$.

Apesar disso, os mecanismos que vinculam a deficiência de zinco ao desenvolvimento cognitivo não estão totalmente claros, pois as análises se dificultam uma vez que a deficiên- 
cia de zinco dificilmente sucede de forma isolada. Parece ser que a deficiência de zinco pode ter como consequência problemas no funcionamento neuropsicológico, nos níveis de atividade e atenção e no desenvolvimento motor, processos todos que interferem no processo de cognição. Nesse contexto, a conjuntura social e o ambiente de cuidados exercem influência importante. Estudo avaliando a influência da suplementação com zinco e o efeito da estimulação psicossocial de crianças encontrou um maior efeito no desenvolvimento nas crianças que foram estimuladas e que receberam a suplementação com zinco, quando comparadas ao grupo controle ou ao grupo que recebeu apenas uma intervenção ${ }^{56}$. Importa destacar, também, as variações segundo idade e sexo (meninos são mais vulneráveis à deficiência de zinco do que as meninas) na relação entre a deficiência de zinco e o desenvolvimento cognitivo ${ }^{52,54}$.

O efeito da suplementação com zinco tem sido testado utilizando dose entre $1-10 \mathrm{mg} / \mathrm{dia}$ durante dois a doze meses, com a aplicação de testes de avaliação do desenvolvimento, incluindo o Bayley Scales of Infant Development (BSID), o Psychomotor Development Index (PDI) e o Mental Development Index (MDI). Apesar de vários estudos mostrarem os efeitos positivos da suplementação com zinco no comportamento e no desenvolvimento motor e cognitivo, os resultados não são consistentes ${ }^{40}$. Estudos adicionais, considerando as características do entorno da população, o nível de deficiência, o tipo de tratamento e sua duração, entre outros fatores, são necessários. Fortes são as evidências sobre o efeito da suplementação com zinco no desempenho neuropsicológico. Não obstante, o desenvolvimento de maior quantidade de pesquisas é necessário para replicar estudos realizados e clarificar o efeito no desempenho acadêmico ${ }^{40,50}$.

\section{Nutrição, crescimento e desenvolvimento}

O processo de crescimento e desenvolvimento é intrinsecamente complexo, pois re- presenta a síntese de uma determinação causal múltipla com a ação de inúmeros fatores endógenos e exógenos. Numerosas investigações têm comprovado a grande influência dos fatores ambientais, destacando o peso decisivo da condição nutricional ${ }^{3}$. No seio desta polêmica, e considerando a determinação comum do crescimento e do desenvolvimento, assume-se a proeminência da associação entre parâmetros nutricionais tanto com o crescimento quanto com o desenvolvimento. As discussões consideradas no presente artigo podem servir de base na tentativa de modelar a determinação simultânea do crescimento e do desenvolvimento pelo fator nutricional.

Pode ser estabelecido um modelo de relações em que a deficiência de micronutrientes específicos poderia afetar o crescimento linear e não afetar necessariamente o desenvolvimento cognitivo. Por exemplo, sabe-se que o ferro tem uma enorme importância para o desenvolvimento cognitivo, mas menor influência no crescimento linear. Isto significa que o crescimento linear e o desenvolvimento cognitivo poderiam não estar correlacionados. A condição anterior deve ocorrer nos casos de inadequação dietética de dois micronutrientes, sendo o primeiro importante para o desenvolvimento e não para o crescimento, e o segundo importante para o crescimento. Sendo assim, considerando o papel do zinco tanto no desenvolvimento cognitivo quanto no crescimento linear, sua deficiência pode representar a forma mais comum na qual o crescimento linear poderia ser utilizado como um bom preditor de desenvolvimento cognitivo. Nesse sentido, importaria saber qual o ponto de corte adequado para indicar o valor a partir do qual a estatura deixa de ter associação direta com o desenvolvimento. Deve esperar-se que a desnutrição afete somente a estatura nas condições de deficiência leve e, talvez, na deficiência moderada. Nas condições de deficiência grave tanto a estatura quanto o desenvolvimento cognitivo deveriam estar afetados e correlacionados. 
O ferro, o zinco e a vitamina A são os micronutrientes que mais limitam o crescimento infantil e o desenvolvimento cognitivo. $\mathrm{O}$ efeito do zinco no crescimento linear dá-se tanto nos casos de deficiência leve como moderada. A suplementação com ferro tem efeito benéfico na estatura de crianças anêmicas e a suplementação com zinco tem impacto significativo, sendo marcante o beneficio em crianças com déficit de estatura. $\mathrm{O}$ ferro e o zinco interferem em funções relacionadas diretamente com o desenvolvimento cognitivo, enquanto o efeito da vitamina A está condicionado pela sua proteção contra as doenças infecciosas.

As deficiências de micronutrientes geralmente manifestam-se de forma conjunta e não existem evidências claras sobre o menor o maior efeito da suplementação com vários micronutrientes quando comparada com a suplementação com um único micronutriente (principalmente o zinco). Sendo, assim, torna-

\section{REFERÊNCIAS}

1. Ministério da Saúde do Brasil. Saúde da criança. Acompanhamento do crescimento e desenvolvimento infantil. Brasília (DF): MS; 2002.

2. Organización Panamericana de la Salud. Manual de crecimiento y desarrollo del niño. Washington (DC): OPS; 1994. (Serie PALTEX para ejecutores de programas de salud; 33).

3. Vasconcelos FAG. Avaliação nutricional de coletividades. $4^{\mathrm{a}}$ ed. Florianópolis: Editora da UFSC; 2008.

4. Uauy R, Kain J, Mericq V, Rojas J, Corvalán C. Nutrition, child growth, and chronic disease prevention. Ann Med 2008; 40(1): 11-20.

5. United Nations, Administrative Committee on Coordination SubCommittee on Nutrition. $4^{\text {th }}$ Report on the World Nutrition Situation: Nutrition se importante o desenvolvimento de pesquisas adicionais nesse contexto.

Não estão claros os mecanismos que vinculam as deficiências de micronutrientes com a capacidade cognitiva. Ensaios clínicos randomizados bem desenhados são de grande importância para esses fins, porém, ressalta-se a problemática metodológica que implica estudar o tema. A grande variedade de fatores que podem afetar o desenvolvimento cognitivo, assim como as recíprocas interações entre os mesmos, constitui um quadro no qual predominam fatores de confusão e de mutuas modificações relacionadas aos seus efeitos no desenvolvimento.

O crescimento e desenvolvimento representam um processo com determinação comum no qual, considerando o peso decisivo da condição nutricional, a deficiência de zinco pode representar a forma mais comum em que o crescimento linear poderia ser utilizado como um bom preditor de desenvolvimento cognitivo.

throughout the life cycle. Ginebra: ACC/ SCN; 2000.

6. Sucupira ACSL, Werner J Jr, Resegue R. Desenvolvimento. In: Sucupira ACSL, Bricks LF, Kobinger MEBA, Saito MI, Zuccoltto SMC, coordenadores. Pediatria em consultório. 4. ${ }^{\text {a }}$ ed. São Paulo: Sarvier; 2000. p. 22-39.

7. Organização Pan-Americana da Saúde. Manual para vigilância do desenvolvimento infantil no contexto da AIDPI. Washington (DC): OPAS; 2005. (Serie OPS/FCH/CA/05.16.P).

8. Singh M. Role of micronutrients for physical growth and mental development. Indian J Pediatr 2004; 71(1): 59-62.

9. Allen LH, Gillespie SR. What works? A review of the efficacy and effectiveness of nutrition interventions. Manila, Philippines: ADB; 2001.

10. Allen LH, Peerson JM, Olney DK. Provision of multiple rather than two or 
fewer micronutrients more effectively improves growth and other outcomes in micronutrient-deficient children and adults. J Nutr 2009; 139(5): 1022-30.

11. Ramakrishnan U, Neufeld LM, Flores R, Rivera J, Martorell R. Multiple micronutrient supplementation during early childhood increases child size at 2 y of age only among high compliers. Am J Clin Nutr 2009; 89(4): 1125-31.

12. Ramakrishnan U, Nguyen P, Martorell R. Effects of micronutrients on growth of children under 5 y of age: meta-analyses of single and multiple nutrient interventions. Am J Clin Nutr 2009; 89: 191-203.

13. Bhutta ZA, Ahmed T, Black RE, Cousens S, Dewey K, Giugliani E, Haider BA, Kirkwood B, Morris SS, Sachdev HP, Shekar M; What works? Interventions for maternal and child undernutrition and survival. Lancet. 2008; 371(9610): 417-40.

14. Chen K, Li TY, Chen L, Qu P, Liu YX. Effects of vitamin A, vitamin A plus iron and multiple micronutrient-fortified seasoning powder on preschool children in a suburb of Chongqing, China. J Nutr Sci Vitaminol 2008; 54(6): 440-7.

15. Lutter CK, Rodríguez A, Fuenmayor G, Avila L, Sempertegui F, Escobar J. Growth and micronutrient status in children receiving a fortified complementary food. J Nutr 2008; 138(2): 379-88.

16. Uauy R, Kain J, Mericq V, Rojas J, Corvalán C. Nutrition, child growth, and chronic disease prevention. Ann Med 2008; 40(1): 11-20.

17. Elizabeth KE, Krishnan V, Zachariah P. Auxologic, biochemical and clinical (ABC) profile of low birth weight babiesa 2-year prospective study. J Trop Pediatr 2007; 53(6): 374-82.

18. Fahmida U, Rumawas JS, Utomo B, Patmonodewo S, Schultink W. Zinc-iron, but not zinc-alone supplementation, increased linear growth of stunted infants with low haemoglobin. Asia Pac J Clin Nutr 2007; 16(2): 301-9.

19. Katz J, Christian P, Dominici F, Zeger SL. Treatment effects of maternal micronutrient supplementation vary by percentiles of the birth weight distribution in rural Nepal. J Nutr 2006; 136(5): 1389-94.

20. Hop le T, Berger J. Multiple micronutrient supplementation improves anemia, micronutrient nutrient status, and growth of Vietnamese infants: double-blind, randomized, placebo-controlled trial. J Nutr 2005; 135(3): 660S-665S.

21. Smuts CM, Dhansay MA, Faber M, Van Stuijvenberg ME, Swanevelder S, Gross R, Benadé AJ. Efficacy of multiple micronutrient supplementation for improving anemia, micronutrient status, and growth in South African infants. J Nutr 2005; 135(3): 653S-659S.

22. Untoro J, Karyadi E, Wibowo L, Erhardt MW, Gross R. Multiple micronutrient supplements improve micronutrient status and anemia but not growth and morbidity of Indonesian infants: a randomized, double-blind, placebo-controlled trial. J Nutr 2005; 135(3): 639S-645S.

23. Christian P, Khatry SK, Katz J, Pradhan EK, LeClerq SC, Shrestha SR, Adhikari RK, Sommer A, West KP Jr. Effects of alternative maternal micronutrient supplements on low birth weight in rural Nepal: double blind randomised community trial. BMJ 2003; 326(7389): 571.

24. Oelofse A, Van Raaij JM, Benade AJ, Dhansay MA, Tolboom JJ, Hautvast JG. The effect of a micronutrient-fortified complementary food on micronutrient status, growth and development of 6- to 12-month-old disadvantaged urban South African infants. Int J Food Sci Nutr 2003; 54(5): 399-407. 
25. Rivera Dommarco J, Hotz C, Gonzalez de Cossío T, Neufeld L, Garcia Guerra A. The effect of micronutrient deficiencies on child growth: a review of results from community-based supplementation trials. Journal Nut 2003; 133(11 Suppl 2): 4010S-20.

26. Bhandari N, Bahl R, Taneja S. Effect of micronutrient supplementation on linear growth of children. Brit J Nut 2001; 85 Suppl 2: S131-37.

27. Dijkhuizen MA, Wieringa FT, West CE, Muherdiyantiningsih M. Concurrent micronutrient deficiencies in lactating mothers and their infants in Indonesia. Am J Clin Nutr 2001; 73(4): 786-91.

28. Wehby GL, Murray JC. The effects of prenatal use of folic acid and other dietary supplements on early child development. Matern Child Health J 2008; 12(2): 180-7.

29. Silva LSV, Thiapó AP, Souza GGS, Saunders C, Ramalho A. Micronutrientes na gestação e lactação. Rev Bras Saúde Matern Infant 2007; 7(3): 237-44.

30. Silva SLV, Thiapó AP, Souza GG de, Saunders C, Ramalho A. Micronutrientes na gestação e lactação. Rev Bras Saúde Matern Infant 2007; 7(3): 237-44.

31. Ministério da Saúde. Carências de micronutrientes. Brasília (DF): MS; 2007.

32. Pinero DJ, Connor JR. Iron in the brain: An important contributor in normal and diseased states. Neuroscientist 2000; 6(6): 435-53.

33. Oppenheimer SJ. Iron and its relation to immunity and infectious disease. J Nutr. 2001; 131(2 Supl 2): 616S-35.

34. Mafra D, Cozzolino SMF. Importância do zinco na nutrição humana. Rev Nutr 2004; 17(1): 79-87.

35. International Zinc Nutrition Consultative Group. Assessment of the risk of zinc deficiency in populations and options for its control. Hotz $\mathrm{C}$ and Brown $\mathrm{KH}$, eds.
Food Nutr Bull 2004; 25(1 Suppl 2): S91204.

36. Black RE. Micronutrients in pregnancy. $\mathrm{Br}$ J Nutr 2001; 85 Suppl 2: S193-7.

37. Llanos A, Uauy R, González de Cossío T, Barros A, para el Proyecto Challenges for Childhood Health and Nutrition Research in Latin America: addressing the 90/10 gap. Bajo Peso al Nacer. Bangladesh: Child Health and Nutrition Research Initiative; 2003.

38. Neufeld L, Hotz C, para el Proyecto Challenges for Childhood Health and Nutrition Research in Latin America: addressing the 90/10 gap. Restricción en el crecimiento y deficiencia de micronutrientes. Bangladesh: Child Health and Nutrition Research Initiative; 2003.

39. Ramakrishnan U, Martorell R. The role of vitamin A in reducing child mortality and morbidity and improving growth. Salud Publica Mex 1998; 40: 189-98.

40. Christa L, Walker F, Black RE. Functional indicators for assessing zinc deficiency. Food Nutr Bull 2007; 28(3 Suppl): S454-79.

41. Chhagan M K, Broeck J V, Luabeya K-K A, Mpontshane N, Tomkins A, Bennish M L. Effect on longitudinal growth and anemia of zinc or multiple micronutrients added to vitamin $\mathrm{A}$ : a randomized controlled trial in children aged 6-24 months. BMC Public Health 2010; 10(145). Disponível em: http:// www.biomedcentral.com/1471-2458/10/ 145. Acesso em: 5 de julho de 2010.

42. Bee H. A criança em desenvolvimento. $9^{\mathrm{a}}$ ed. Porto Alegre: Artmed; 2003.

43. Fernandes A. Nutrição e Desenvolvimento. In: Silva AC, GomesPedro J, editors. Nutrição Pediátrica: Princípios Básicos. Lisboa: ACSM; 2005. p. 225-33.

44. Sachdev HPS, Gera T, Nestel P. Effect of iron supplementation on mental and motor development in children: systematic review 
of randomised controlled trials. Public Health Nutrition 2004; 8(2): 117-32.

45. Guardiola A, Egewarth C, Rotta NT. Avaliação do desenvolvimento neuropsicomotor em escolares de primeira série e sua relação com o estado nutricional. J Pediatr 2001; 77(3): 189-96.

46. Santos-Monteiro J, Guedes RCA, Castro RM, Filho JEC. Psychosocial stimulation and brain plasticity in malnourished individuals. Rev Bras Saude Matern Infant 2002; 2(1): 15-22.

47. Fernandes A. Nutrição e desenvolvimento. Nutrição Pediátrica: Princípios Básicos. Lisboa: ACSM; 2005.

48. Lozoff B. Iron deficiency and child development. Food Nutr Bull 2007; 28 (4 Suppl): S560-71.

49. Black MM. Micronutrient Deficiencies and Cognitive Functioning. J. Nutr 2003; 133 Suppl: 3927S-31.

50. Grantham-McGregor S, Ani CC. The role of micronutrients in psychomotor and cognitive development. British Medical Bulletin 1999; 55(3): 511-27.

51. Humphrey JH, Agoestina T, Juliana A, Septiana S, Widjaja H, Cerreto MC, Wu Lee SF, Ichord RN, Katz J, and West KP. Neonatal vitamin A supplementation: effect on development and growth at $3 \mathrm{y}$ of age. Am J Clin Nutr 1998; 68: 109-17.

52. Sandstead HH, Penland JG, Alcock NW, Dayal HH, Xue CCh, Jui SL, Faji Z, Jia JY. Effects of repletion with zinc and other micronutrients on neuropsychologic performance and growth of Chinese children. Am J Clin Nutr 2007; 86: 412-20.

53. Rosado JL. Deficiencia de zinc y sus implicaciones funcionales. Salud Pública Mex 1998; 40(2): 181-8.

54. Black MM. Zinc deficiency and child development. Am J Clin Nutr 1998; 68 Suppl: 464S-9.

55. Salgueiro MJ, Weill R, Hernándezb-Triana M, Zubillaga M, Lysionek A, Caro R. Deficiência de zinc em relación com el desarrollo intelectual y sexual. Rev Cubana Salud Pública 2004; 30(2). Disponível em: http://scielo.sld.cu/ scielo.php?script $=$ sci_arttext\&pid $=$ S08644662004000200007\&lng=es. Acesso em: 5 de julho de 2010.

56. Gardner JMM, Powell CA, BakerHenningham H, Walker SP, Cole TJ. Zinc supplementation and psychosocial stimulation: effects on the development of undernourished Jamaican children. Am J Clin Nutr 2005; 82: 399-405

Recebido em: 19/mai./10 Modificado em 18/dez./10 Aprovado em 28/dez./10 